\title{
Lagrangian duality and cone convexlike functions
}

\author{
J.B.G. Frenk and G. Kassay
}

\begin{tabular}{|l|l|}
\hline \multicolumn{2}{|l|}{ ERIM REPORT SERIES RESEARCH IN MANAGEMENT } \\
\hline ERIM Report Series reference number & ERS-2005-019-LIS \\
\hline Publication & March 2005 \\
\hline Number of pages & 18 \\
\hline Email address corresponding author & frenk@few.eur.nl \\
\hline Address & Erasmus Research Institute of Management (ERIM) \\
& Rotterdam School of Management / Rotterdam School of Economics \\
& Erasmus Universiteit Rotterdam \\
& P.O.Box 1738 \\
& 3000 DR Rotterdam, The Netherlands \\
& Phone: $\quad+31104081182$ \\
& Fax: $\quad+31104089640$ \\
& Email: info@erim.eur.nl \\
& Internet: $\quad$ www.erim.eur.nl \\
\hline
\end{tabular}

Bibliographic data and classifications of all the ERIM reports are also available on the ERIM website: www.erim.eur.nl 


\title{
ERASMUS RESEARCH INSTITUTE OF MANAGEMENT
}

\section{REPORT SERIES}

\author{
RESEARCH IN MANAGEMENT
}

\begin{tabular}{|c|c|}
\hline \multicolumn{2}{|c|}{ ABSTRACT AND KEYWORDS } \\
\hline Abstract & $\begin{array}{l}\text { In this paper we will show that the closely K-convexlike vector-valued functions with } \\
\mathrm{K} \mathrm{R} \mathrm{R}^{\mathrm{m}} \text { a nonempty convex cone and related classes of vector-valued functions discussed } \\
\text { in the literature arise naturally within the theory of biconjugate functions applied to the } \\
\text { Lagrangian perturbation scheme in finite dimensional optimization. For these classes of } \\
\text { vectorvalued functions an equivalent characterization of the dual objective function associated } \\
\text { with the Lagrangian is derived by means of a dual representation of the relative interior of a } \\
\text { convex cone. It turns out that these characterizations are strongly related to the closely } \\
\text { convexlike and Ky-Fan convex bifunctions occurring within minimax problems. Also it is shown } \\
\text { for a general class of finite dimensional optimization problems that strong Lagrangian duality } \\
\text { holds in case a vector-valued function related to the functions in this optimization problem is } \\
\text { closely K-convexlike and satisfies some additional regularity condition. }\end{array}$ \\
\hline Free Keywords & Lagrangian duality, cone convexlike functions \\
\hline
\end{tabular}




\title{
Lagrangian duality and cone convexlike functions
}

\author{
J.B.G. Frenk* \\ G. Kassay ${ }^{\dagger}$
}

March 15, 2005

\begin{abstract}
In this paper we will show that the closely $K$-convexlike vector-valued functions with $K \subseteq \mathbb{R}^{m}$ a nonempty convex cone and related classes of vector-valued functions discussed in the literature arise naturally within the theory of biconjugate functions applied to the Lagrangian perturbation scheme in finite dimensional optimization. For these classes of vectorvalued functions an equivalent characterization of the dual objective function associated with the Lagrangian is derived by means of a dual representation of the relative interior of a convex cone. It turns out that these characterizations are strongly related to the closely convexlike and Ky-Fan convex bifunctions occurring within minimax problems. Also it is shown for a general class of finite dimensional optimization problems that strong Lagrangian duality holds in case a vector-valued function related to the functions in this optimization problem is closely $K$-convexlike and satisfies some additional regularity condition.
\end{abstract}

\section{Introduction.}

For $D$ a nonempty set of a vector space $X$ let $f: D \rightarrow \mathbb{R}$ be some real-valued function and $F: D \rightarrow \mathbb{R}^{m}$ some vector-valued function. Consider now the optimization problem

$$
v(P):=\inf \{f(x): x \in \mathcal{F}\}
$$

with nonempty feasible region $\mathcal{F}:=\{x \in D: F(x) \in-K\}$ and $K \subseteq \mathbb{R}^{m}$ a proper convex cone. It is well-known that one can associate with the above so-called primal problem $(P)$ the Lagrangian dual optimization problem $(D)$ given by

$$
v(D):=\sup \left\{\theta(\lambda): \lambda \in K^{*}\right\} .
$$

In the optimization problem $(D)$ the nonempty set $K^{*}:=\left\{\lambda \in \mathbb{R}^{m}: \lambda^{T} y \geq 0\right.$ for every $\left.y \in K\right\}$ denotes the dual cone of $K$ and $\theta: K^{*} \rightarrow[-\infty, \infty)$ is the Lagrangian function given by

$$
\theta(\lambda):=\inf \left\{f(x)+\lambda^{\top} F(x): x \in D\right\}
$$

It can be shown relatively easy that $v(P) \geq v(D)$ (weak duality) and an important theoretical issue in optimization theory is to determine under which conditions on the function pair $(F, f)$ one

${ }^{*}$ J.B.G.Frenk, Econometric Institute, Erasmus University, P.O. Box 1738, 3000DR Rotterdam, The Netherlands, Email: frenk@few.eur.nl

${ }^{\dagger}$ Corresponding author: G.Kassay, Department of Mathematics, Eastern Mediterranean University, Mersin 10, Gazimağusa, TRNC, Turkey, E-mail: gabor.kassay@emu.edu.tr 
actually has that $v(D)$ equals $v(P)$ and additionally the dual problem $(D)$ has an optimal solution. It is well-known (cf.[17]) that this holds under convexity conditions on $(F, f)$ in combination with some regularity assumption on the feasible region $\mathcal{F}$ known as the generalized Slater condition. These conditions are sufficient and imply that the value function $p$ of the Lagrangian perturbation problem with perturbation space $Y$ (cf.[17], [18]) associated with the optimization problem $(P)$ is a convex function on $Y$. By generalizing in the first part of Section 3 some classical convexity results used within this perturbation approach it is shown in the second part of Section 3 that strong Lagrangian duality holds under weaker sufficient conditions on $p$. In particular, strong Lagrangian duality holds if the lower semicontinuous hull $\bar{p}$ of $p$ is convex in combination with some regularity conditions. Also it is verified that the function $\bar{p}$ is convex if and only if some vectorvalued function related to the function pair $(F, f)$ is closely convexlike. By these observations the connection between cone convexlike vector-valued functions and Lagrangian strong duality is established.. Due to this we start in Section 2 with an overview on some of the different cone convexlike vector-valued functions discussed in the literature (see for example [21], [9], [10], [16], [13], [1], [5], [14]). Observe these functions are given various names and in most of the literature (contrary to this paper and [5]) it is assumed that the associated cone has a nonempty interior. At the end of Section 2 we also translate the different cone convexlike properties of vector-valued functions to properties of real-valued bifunctions. In particular, for $r i(K)$-convexlike and closely $K$-convexlike vector-valued functions these results seem to be new. Finally, in Section 3 we first extend some of the classical convexity results known within the literature (see for example [17] or [6]) and, as already mentioned, apply these results in the second part of this section to the Lagrangian perturbation scheme of Rockafellar. In particular, we will derive strong Lagrangian duality results under weaker cone convexlike properties of some vector-valued function related to the primal optimization problem $(P)$.

Instead of the perturbation approach of Rockafellar to derive Lagrangian duality results one could also have used the so-called image space approach first considered by Giannessi (cf.[8]). In this approach one shows that the intersection of a convex set and a certain convex conic extension of the image of a vector-valued function related to the original optimization problem $(P)$ is empty. Such an approach can be found in the already mentioned paper of Giannessi and for a recent example of this approach to so-called parametric generalized systems the reader is referred to [14]. Observe in [14] a general parametric system is considered where the image space is a topological linear space. Using the image space approach it is shown that under certain cone convexity-type conditions these systems satisfy a theorem of the alternative. It is well known that the Lagrangian duality model can also be put into this framework (cf.[5]) and so it is in principle possible to derive similar results using the image space approach. However, in [14] the authors do not consider the finite dimensional case in detail. In particular, by the infinite dimensionality of the image space it is assumed in [14] that the cone under consideration has a nonempty interior. In this paper the

image space is finite dimensional and this means that we do not need to assume that the cone under consideration has a nonempty interior. This enables us to derive stronger results for our particular case.

\section{On cone convexlike vector-valued functions.}

In this section we first discuss some elementary properties of the sets $S+K$ with $K \subseteq \mathbb{R}^{m}$ a nonempty proper convex cone and $S \subseteq \mathbb{R}^{m}$ arbitrary. These properties will be used at the end of this section to discuss relations between various known classes of cone convexlike vector-valued 
functions. Also we will investigate the relation between cone convexlike vector-valued functions and several classes of real-valued bifunctions considered within the minimax literature (cf.[7]). To start with our discussion we first recall some well-known concepts. A nonempty set $K \subseteq \mathbb{R}^{m}$ is called a cone if $\alpha K \subseteq K$ for every $\alpha>0$ and it is called convex if $\alpha K+(1-\alpha) K \subseteq K$ for every $0<\alpha<1$ (cf.[17], [6]). Contrary to some authors it is not assumed that the zero element belongs to a cone. We now list some elementary properties of the sets $S+K$. Since for a convex set $K$ it holds that $\alpha K+(1-\alpha) K=K$ for every $0<\alpha<1$, it is obvious that

$$
S+K \text { convex } \Longleftrightarrow \alpha S+(1-\alpha) S+K \subseteq S+K \text { for } 0<\alpha<1 .
$$

Also it is well-known (cf.[17]) that the sets $r i(K)$ and $c l(K)$ are again nonempty convex cones, where $r i$ denotes the relative interior and $c l$ the closure operator. This implies that relation (1) also holds with $K$ replaced by $r i(K)$ or $c l(K)$. To verify an equivalent characterization of $c l(S+K)$ convex we first observe (cf.[5]) that $c l(S+c l(K))=c l(S+r i(K))$. This shows

$$
c l(S+\operatorname{cl}(K))=\operatorname{cl}(S+K)=\operatorname{cl}(S+\operatorname{ri}(K)) .
$$

Applying the first equality in relation (2) and $\mathbf{0} \in \operatorname{cl}(K)$, it follows that $S \subseteq \operatorname{cl}(S+K)$. Also for $c l(S+K)$ convex it is obvious that $\alpha(S+K)+(1-\alpha)(S+K) \subseteq c l(S+K)$ for every $0<\alpha<1$. Using these two observations one can show by standard arguments that

$$
c l(S+K) \text { convex } \Longleftrightarrow \alpha S+(1-\alpha) S \subseteq c l(S+K) \text { for } 0<\alpha<1 .
$$

Moreover, by relations (1) and (2) the following inclusions are easy to show (cf.[5], [14])

$$
S+K \text { convex } \Rightarrow S+\operatorname{ri}(K) \text { convex } \Rightarrow \operatorname{cl}(S+K) \text { convex. }
$$

For arbitrary convex cones $K \subseteq \mathbb{R}^{m}$ the implications in relation (4) are strict (cf.[5]). However, under some additional assumption on the sets $S$ and $K$ one can show that the last implication in relation (4) can actually be reversed. To prove this we need to verify a technical result. This is shown in the Appendix. Recall that the set $\operatorname{lin}(S)$ represents the linear hull of $S$, while the set aff $f(S)$ denotes the affine hull of $S$ (cf.[6]).

Lemma 1 For $K \subseteq \mathbb{R}^{m}$ a nonempty convex cone and $S \subseteq \mathbb{R}^{m}$ a set satisfying $S \subseteq x_{0}+\operatorname{lin}(K)$ for some $x_{0} \in$ af $f(S)$, it follows

$$
S+\operatorname{ri}(K) \text { convex } \Longleftrightarrow c l(S+K) \text { convex. }
$$

Proof. By relation (4) we only have to verify that $c l(S+K)$ convex implies $S+r i(K)$ convex. By Theorem 27 it follows that $\operatorname{ri}(\operatorname{cl}(S+K))=S+\operatorname{ri}(K)$, and since the relative interior of a convex set is again convex, the result follows.

In the definition of a cone we did not assume that the zero element belongs to this cone. However, in many specific cones occurring within finite dimensional optimization the zero element is included. It is now natural to investigate whether this additional assumption enables us to improve the above results. If we consider relation (1) this is indeed the case and we obtain

$$
S+K \text { convex } \Longleftrightarrow \alpha S+(1-\alpha) S \subseteq S+K \text { for } 0<\alpha<1
$$

for any convex cone $K$ containing $\mathbf{0}$. Although relation (5) is listed for convex cones belonging to $\mathbb{R}^{m}$ one can verify the same relation by exactly the same proof for cones belonging to a vector 
space $X$. This observation will be useful in verifying relation (11) below. As shown by the following example the condition $\mathbf{0} \in K$ is essential in relation (5). Note that $\mathbb{R}_{++}^{m}:=\operatorname{int}\left(\mathbb{R}_{+}^{m}\right)$ for any $m \in \mathbb{N}$ with int denoting the interior and $\mathbb{R}_{+}^{m}$ the positive orthant of $\mathbb{R}^{m}$.

Example 2 Consider the convex set $S=[0,2] \times\{0\} \subseteq \mathbb{R}^{2}$. For this set it follows that $S+\mathbb{R}_{++}^{2}$ equals $\mathbb{R}_{++}^{2}$ and for every $0<\alpha<1$ the set $\alpha S+(1-\alpha) S=S$ is not included in $S+\mathbb{R}_{++}^{2}$. This shows that the condition $\mathbf{0} \in K$ cannot be omitted in relation (5).

If $K$ is a convex cone containing $\mathbf{0}$ one can show the following improvement of relation (4).

Lemma 3 For $K \subseteq \mathbb{R}^{m}$ a convex cone containing 0 and $S \subseteq \mathbb{R}^{m}$ it follows

$$
S+K \text { convex } \Rightarrow S+\operatorname{cl}(K) \text { convex } \Rightarrow S+\text { ri }(K) \text { convex. }
$$

Proof. If $S+K$ is convex and $\mathbf{0} \in K$, it follows by relation (5) that $\alpha S+(1-\alpha) S \subseteq S+K \subseteq$ $S+\operatorname{cl}(K)$ for every $0<\alpha<1$. This implies again by relation (5) that $S+\operatorname{cl}(K)$ is convex. If $S+\operatorname{cl}(K)$ is convex, then similarly $\alpha S+(1-\alpha) S \subseteq S+c l(K)$ and using $c l(K)+\operatorname{ri}(K) \subseteq \operatorname{ri}(K)$ we obtain

$$
\alpha S+(1-\alpha) S+r i(K) \subseteq S+c l(K)+r i(K) \subseteq S+r i(K)
$$

for every $0<\alpha<1$. Applying the observation after relation (1) yields $S+\operatorname{ri}(K)$ is convex.

The following example shows that in general the first and second implication in Lemma 3 are strict implications.

Example 4 To show that the first implication of Lemma 3 is strict let $K:=\mathbb{R}_{++}^{2} \cup\{\mathbf{0}\}$ and $S:=\{(1,0),(2,0)\}$. For these sets it follows that $S+K$ is not convex and $S+c l(K)$ is convex. To verify that the second implication of Lemma 3 is strict, let $K:=\{(s, t): t \geq s \geq 0\}$ and $S:=\{(s, 0): s \in \mathbb{Q}\}$. For these sets we obtain that $S+\operatorname{ri}(K)$ is convex, while $S+\operatorname{cl}(K)$ is not convex.

Within the considered chain of implications it is clear that the improvement of Lemma 3 over relation (4) is given by the intermediate result $S+\operatorname{cl}(K)$ convex. It is now natural to ask whether $S+\operatorname{cl}(K)$ convex also holds without the additional assumption that $K$ contains $\mathbf{0}$. The first part of the following example yields a negative answer. Similarly, one can present a convex cone $K$ not containing $\mathbf{0}$ satisfying $S+\operatorname{cl}(K)$ is convex, while $S+K$ is not convex. This shows in particular for convex cones $K$ not containing 0 that the properties $S+K$ convex and $S+c l(K)$ convex are not related.

Example 5 To show an example of a set $S$ and a convex cone $K$ not containing 0 satisfying $S+K$ is convex and $S+\operatorname{cl}(K)$ is not convex, let $K:=\{(s, t): t>s>0\}$ and $S:=\{(s, 0): s \in \mathbb{Q}\}$. Clearly 0 does not belong to $K$ and the set $S+K$ is convex, while the set $S+c l(K)$ is not convex. To show an example of a set $S$ and a convex cone $K$ not containing 0 satisfying $S+K$ is not convex and $S+c l(K)$ is convex let $K:=\{(s, 0,0): s>0\} \cup\{(s, t, u): u>0\}$ and $S:=\{(0,0,0),(0,1,0)\}$. It is easy to verify that $K$ is a convex cone and 0 does not belong to $K$. Since $(1,0,0)$ and $(1,1,0)$ do belong to $S+K$ but $\left(1, \frac{1}{2}, 0\right)$ does not, the set $S+K$ is not convex. The set $\operatorname{cl}(K)$ is now given by $\{(s, u, t): u \geq 0\}$ and so $S+\operatorname{cl}(K)=\operatorname{cl}(K)$ showing that $S+\operatorname{cl}(K)$ is convex. 
We will now apply the above elementary results for sets $S+K$ to the different classes of cone convexlike vector-valued functions discussed in the literature (for a survey see [5]). Recall for a nonempty proper convex cone $K$ that the transitive ordering $y \leq_{K} x$ is defined by $x-y \in K$. Note that this ordering is a partial ordering (cf.[19]) if the cone $K$ is pointed, i.e $K \cap-K=\{0\}$. The epigraph epi $\sin _{K}(F)$ of a vector-valued function $F: D \rightarrow \mathbb{R}^{m}$, where $D$ is a subset of a vector space $X$, with respect to this ordering is given by

$$
\operatorname{epi}_{K}(F):=\left\{(x, r): x \in D, F(x) \leq_{K} r\right\} \subseteq D \times \mathbb{R}^{m} .
$$

To list some useful relations we introduce the function $F_{0}: D \rightarrow D \times \mathbb{R}^{m}$ given by $F_{0}(x):=$ $(x, F(x))$ and associate with the convex cone $K \subseteq \mathbb{R}^{m}$ the convex cone $K_{0}:=\left\{\mathbf{0}_{X}\right\} \times K \subseteq$ $X \times \mathbb{R}^{m}$ with $\mathbf{0}_{X}$ denoting the zero element of the vector space $X$. It is easy to see that

$$
\operatorname{epi}_{K}(F)=F_{0}(D)+K_{0},
$$

with $G(D):=\{G(x): x \in D\}$ the range of a vector-valued function $G$. Moreover, for the projection $A: D \times \mathbb{R}^{m} \rightarrow \mathbb{R}^{m}$ given by $A(x, r)=r$ it follows that

$$
A\left(\operatorname{epi}_{K}(F)\right)=F(D)+K .
$$

The next definition introduces some classes of cone convexlike vector-valued functions used within the literature (see for example [5] or [14]).

Definition 6 Let $K \subseteq \mathbb{R}^{m}$ be a nonempty convex cone. The vector-valued function $F: D \rightarrow \mathbb{R}^{m}$ is called $K$-convex if the set epi $i_{K}(F)$ is convex. It is called $K$-convexlike, respectively closely $K$-convexlike, if the set $F(D)+K$, respectively $\operatorname{cl}(F(D)+K)$ is convex.

For each of the above function classes it is easy to show that they are closed under addition and multiplication with a positive scalar. This means that each class of functions is a convex cone itself. In the literature a $r i(K)$-convexlike function is also called $K$-subconvexlike (cf.[10], [5], [1]). To show the implications of the results derived for the sets $S+K$ with $K$ a nonempty convex cone we first consider the class of $K$-convex functions. Since for any convex cone $K \subseteq \mathbb{R}^{m}$ it is well-known that $\operatorname{ri}\left(K_{0}\right)=\operatorname{ri}\left(\left\{\mathbf{0}_{X}\right\} \times K\right)=\left\{\mathbf{0}_{X}\right\} \times r i(K)$ and $\operatorname{ri}(K)$ is a nonempty convex cone, we obtain by relation (6) and the first implication in relation (4) that

$$
F \text { is } K \text {-convex } \Rightarrow F \text { is } r i(K) \text {-convex. }
$$

In the next example it is shown that the set of $K$-convex functions is strictly included in the set of $r i(K)$-convex functions.

Example 7 Let $K=\mathbb{R}_{++}^{2} \cup\{\mathbf{0}\}$ and consider the vector-valued function $F: \mathbb{R}^{2} \rightarrow \mathbb{R}^{2}$ given by

$$
F(x):= \begin{cases}(0,0) & \text { for } x \in \mathbb{R} \times \mathbb{R}_{+} \\ (1,0) & \text { otherwise. }\end{cases}
$$

It is clear that epi $i_{r i(K)}(F)=\mathbb{R}^{2} \times \mathbb{R}_{+}^{2}$ and so $F$ is ri $(K)$-convex. Suppose now that $F$ is also $K$-convex and consider the vectors $\left(x_{i}, r_{i}\right) \in \mathbb{R}^{4}, i=1,2$ with $x_{1}:=(1,4), x_{2}:=(1,-2)$, $r_{1}:=(0,0)$ and $r_{2}=(1,0)$. Since 0 belongs to $K$ we obtain that both vectors belong to epi $i_{K}(F)$ and this implies by the convexity of epi $i_{K}(F)$ that

$$
2^{-1}\left(x_{1}, r_{1}\right)+2^{-1}\left(x_{2}, r_{2}\right)=\left(1,1,2^{-1}, 0\right) \in \operatorname{epi}_{K}(F) .
$$

By relation (9) it follows that $\left(2^{-1}, 0\right) \in K+F(1,1)=K$ and we obtain a contradiction. Hence $F$ is not $K$-convex. 
In Example 7 the cone $K$ is not closed. However, for $K$ closed one can give an improvement of relation (8). As shown by the next theorem the sets of $r i(K)$-convex and $K$-convex functions coincide for $K$ a closed convex cone.

Theorem 8 For $F: D \rightarrow \mathbb{R}^{m}$ a vector-valued function and $K \subseteq \mathbb{R}^{m}$ a closed convex cone it follows that

$$
F \text { is } K \text {-convex } \Longleftrightarrow F \text { is ri }(K) \text {-convex. }
$$

Proof. By relation (8) we have to verify that any $r i(K)$-convex function is also $K$-convex. Suppose the vector-valued function $F$ is $r i(K)$-convex and consider some $\left(x_{i}, r_{i}\right) \in \operatorname{epi}_{K}(F), i=1,2$. This implies by definition that $r_{i}-F\left(x_{i}\right) \in K$ and for a fixed $k_{0} \in r i(K)$ and arbitrary $n \in N$ we obtain

$$
r_{i}+n^{-1} k_{0}-F\left(x_{i}\right) \in \operatorname{cl}(K)+n^{-1} k_{0} \subseteq \operatorname{ri}(K) .
$$

This shows that the vector $\left(x_{i}, r_{i}+n^{-1} k_{0}\right), i=1,2$ belongs to epi $\mathrm{i}_{\mathrm{ri}(K)}(F)$ and since epi $\mathrm{ri}_{\mathrm{ri}(K)}(F)$ is convex it follows for every $0<\alpha<1$ that $\alpha x_{1}+(1-\alpha) x_{2} \in D$ and

$$
\alpha r_{1}+(1-\alpha) r_{2}+n^{-1} k_{0}-F\left(\alpha x_{1}+(1-\alpha) x_{2}\right) \in \operatorname{ri}(K) .
$$

Letting $n \uparrow \infty$ in relation (10) we obtain

$$
\alpha r_{1}+(1-\alpha) r_{2}+n^{-1} k_{0}-F\left(\alpha x_{1}+(1-\alpha) x_{2}\right) \in \operatorname{cl}(r i(K)) .
$$

This shows using $\operatorname{cl}(\operatorname{ri}(K))=\operatorname{cl}(K)=K$ and $\alpha x_{1}+(1-\alpha) x_{2} \in D$ that the set $\operatorname{epi}_{K}(F)$ is convex.

As already observed, it is mostly assumed in the literature (see for example [2]) that the convex cone $K$ contains $\mathbf{0}$. If this additional condition holds another equivalent definition of a $K$-convex function can be given. This definition is frequently encountered within the literature (see for example [2]) and resembles the classical definition of a convex function. Applying relation (5) and (6) yields

$$
F \text { is } K \text {-convex } \Longleftrightarrow D \text { is convex and } F\left(\alpha x_{1}+(1-\alpha) x_{2}\right) \leq_{K} \alpha F\left(x_{1}\right)+(1-\alpha) F\left(x_{2}\right)
$$

for any convex cone $K$ containing $\mathbf{0}$. Next we discuss convexlike functions with respect to $K$. We observe by relation (1) that

$$
F \text { is } K \text {-convexlike } \Longleftrightarrow \alpha F(D)+(1-\alpha) F(D)+K \subseteq F(D)+K
$$

for every $0<\alpha<1$. If additionally the zero element belongs to $K$ it follows by relation (5) that

$$
F \text { is } K \text {-convexlike } \Longleftrightarrow \alpha F(D)+(1-\alpha) F(D) \subseteq F(D)+K
$$

for every $0<\alpha<1$. Similarly by the observation after relation (1) we obtain

$$
F \text { is } r i(K) \text {-convexlike } \Longleftrightarrow F(D)+(1-\alpha) F(D)+r i(K) \subseteq F(D)+r i(K)
$$

for every $0<\alpha<1$ and by relation (3) that

$$
F \text { is closely } K \text {-convexlike } \Longleftrightarrow \alpha F(D)+(1-\alpha) F(D) \subseteq \operatorname{cl}(F(D)+K)
$$


for every $0<\alpha<1$. Finally we obtain by relations (4) and (7) that the following implications hold (cv denotes an abbreviation for convex and the symbols stand for the whole class of functions having the corresponding property)

$K$-cv $\subseteq K$-cvlike $\subseteq$ ri $(K)$-cvlike $\subseteq$ closely $K$-cvlike.

It can be shown (cf.[5]) that the above inclusions are strict. Finally, we mention for $K \subseteq \mathbb{R}^{m}$ and $F: D \rightarrow \mathbb{R}^{m}$ satisfying $F(D) \subseteq x_{0}+\operatorname{lin}(K)$ for some $x_{0} \in \operatorname{aff}(F(D))$ (sufficient condition: $K$ has a nonempty interior) that by Theorem 27

$$
F \text { is } r i(K) \text {-convexlike } \Longleftrightarrow F \text { is closely } K \text {-convexlike. }
$$

Using the above relations and duality results for convex cones one can translate some of the above properties of vector-valued functions to real-valued bifunctions. The first duality result needed for this translation is the well-known bipolar theorem $\operatorname{cl}(K)=K^{* *}$ with $K^{*}:=\left\{\lambda \in \mathbb{R}^{m}: \lambda^{\top} x \geq 0\right.$ for every $x \in K\}$ the dual cone of $K$ and $K^{* *}:=\left(K^{*}\right)^{*}$. Also we need a refinement of the bipolar theorem for relatively open convex cones given by (cf.[20])

$$
x \in \operatorname{ri}(K) \Longleftrightarrow x \in \operatorname{lin}(K) \text { and } \lambda^{\top} x>0 \text { for } \lambda \in K^{*} \backslash K^{\perp}
$$

with $K^{\perp}:=\left\{\lambda \in \mathbb{R}^{m}: \lambda^{\top} x=0\right.$ for every $\left.x \in K\right\}$ denoting the orthogonal complement of $K$. To give an equivalent characterization of relatively open convex cones, which can be applied directly within the proof of the next theorems, we observe using $\operatorname{lin}(K)=\left(K^{\perp}\right)^{\perp}$ (cf.[6]) that $\operatorname{lin}(K) \cap K^{\perp}=\{\boldsymbol{0}\}$. This shows

$$
\left(K^{*} \cap \operatorname{lin}(K)\right) \backslash\{\mathbf{0}\} \subseteq K^{*} \backslash K^{\perp} .
$$

Moreover, by the orthogonal projection theorem (cf.[11]) it follows for every $\lambda \in \mathbb{R}^{m}$ and $x \in$ $\operatorname{lin}(K)$ that $\lambda^{\top} x=\lambda_{1}^{\top} x$ with $\lambda_{1} \in \operatorname{lin}(K)$ denoting the orthogonal projection of $\lambda$ onto the linear subspace $\operatorname{lin}(K)$. Using these observations it follows for $x \in \operatorname{lin}(K)$ that

$$
\lambda^{\top} x>0 \text { for } \lambda \in K^{*} \backslash K^{\perp} \Longleftrightarrow \lambda^{\top} x>0 \text { for } \lambda \in\left(K^{*} \cap \operatorname{lin}(K)\right) \backslash\{\mathbf{0}\} .
$$

Hence by relations (17), (18) and (19) an equivalent dual characterization of relatively open convex cones is given by

$$
x \in \operatorname{ri}(K) \Longleftrightarrow x \in \operatorname{lin}(K) \text { and } \lambda^{\top} x>0 \text { for } \lambda \in\left(K^{*} \cap \operatorname{lin}(K)\right) \backslash\{\mathbf{0}\} .
$$

In the next definition we introduce a class of functions well-known within the minimax literature (cf.[3], [7]).

Definition 9 The bifunction $f: K^{*} \times D \rightarrow \mathbb{R}$ is called convex on $D$ if the set $D$ is convex, and for every $x_{1}, x_{2} \in D$ and $0<\alpha<1$ it follows that

$$
f\left(\lambda, \alpha x_{1}+(1-\alpha) x_{2}\right) \leq \alpha f\left(\lambda, x_{1}\right)+(1-\alpha) f\left(\lambda, x_{2}\right)
$$

for every $\lambda \in K^{*}$. The bifunction $f: K^{*} \times D \rightarrow \mathbb{R}$ is called Ky-Fan convex on $D$ if for every $x_{1}, x_{2} \in D$ and $0<\alpha<1$ there exists some $x_{0} \in D$ satisfying

$$
f\left(\lambda, x_{0}\right) \leq \alpha f\left(\lambda, x_{1}\right)+(1-\alpha) f\left(\lambda, x_{2}\right)
$$

for every $\lambda \in K^{*}$. 
The next result is well-known and a direct consequence of the bipolar theorem and relations (13) and (6) (see for example [2] or [5]). In Theorems 10, 12 and 14 below the bifunction $f$ : $K^{*} \times D \rightarrow \mathbb{R}$ has the special form

$$
f(\lambda, x)=\lambda^{\top} F(x)
$$

with $F: D \rightarrow \mathbb{R}^{m}$ some vector-valued function.

Theorem 10 For $K \subseteq \mathbb{R}^{m}$ a nonempty convex cone it follows that the vector-valued function $F: D \rightarrow \mathbb{R}^{m}$ is cl( $\left.K\right)$-convex on $D$ if and only if the function $f: K^{*} \times D \rightarrow \mathbb{R}$ defined in (21) is convex on $D$. Moreover, the vector-valued function $F: D \rightarrow \mathbb{R}^{m}$ is cl $(K)$-convexlike if and only if the function $f: K^{*} \times D \rightarrow \mathbb{R}$ defined in (21) is Ky-Fan convex on $D$.

By Theorem 8 we know that $F$ is $c l(K)$-convex if and only if $F$ is $\operatorname{ri}(K)$-convex and this shows that the dual representation for $\mathrm{cl}(K)$-convex functions in Theorem 10 also holds for $\mathrm{ri}(K)$ convex functions. Unfortunately, since for arbitrary convex cones $K \subseteq \mathbb{R}^{m}$ it is not possible to give a dual characterization of $K$, it seems difficult to give a dual representation of a $K$-convex function. A similar observation also holds for $K$-convexlike functions. On the other hand, since in relation (17) a dual representation for relatively open convex cones is given, it is possible to give a dual representation for a $r i(K)$-convexlike and a closely $K$-convexlike function. To define the proper class of bifunctions for $\mathrm{ri}(K)$-convexlike functions we introduce the following definition.

Definition 11 The bifunction $f: K^{*} \times D \rightarrow \mathbb{R}$ belongs to the class $\mathcal{A}$ iffor every $\epsilon>0,0<\alpha<1$ and $x_{1}, x_{2} \in D$ there exists some $x_{0} \in D$ such that

$$
f\left(\lambda, x_{0}\right) \leq \alpha f\left(\lambda, x_{1}\right)+(1-\alpha) f\left(\lambda, x_{2}\right)+\epsilon\|\lambda\|
$$

for every $\lambda \in P_{1}$ and

$$
f\left(\lambda, x_{0}\right)=\alpha f\left(\lambda, x_{1}\right)+(1-\alpha) f\left(\lambda, x_{2}\right)
$$

for every $\lambda \in P_{2}$ with the sets $P_{1}, P_{2}$ a partition of $K^{*}$.

In the next theorem we derive a dual characterization of a $r i(K)$-convexlike function. Note that the set $B$ denotes the closed Euclidean unit ball in $\mathbb{R}^{m}$ and so its boundary $\partial B$ is given by $\partial B=\{\lambda:\|\lambda\|=1\}$.

Theorem 12 For $K \subseteq \mathbb{R}^{m}$ a nonempty convex cone it follows that $F: D \rightarrow \mathbb{R}^{m}$ is ri(K)convexlike if and only if the function $f: K^{*} \times D \rightarrow \mathbb{R}$ defined in (21) belongs to the set $\mathcal{A}$ with $P_{1}=K^{*} \backslash K^{\perp}$ and $P_{2}=K^{\perp}$.

Proof. Let $F$ be a $\operatorname{ri}(K)$-convexlike function and consider some arbitrary $\epsilon>0,0<\alpha<1$ and $x_{1}, x_{2} \in D$. Since the set $r i(K)$ is a nonempty cone one can find some $k_{0} \in \operatorname{ri}(K) \cap \epsilon B$. This implies by relation (14) that there exists some $x_{0} \in D$ satisfying

$$
\alpha F\left(x_{1}\right)+(1-\alpha) F\left(x_{2}\right)-F\left(x_{0}\right)+k_{0} \in \operatorname{ri}(K) .
$$

Applying the dual representation of $r i(K)$ given by (17), the Cauchy-Schwarz inequality and $\left\|k_{0}\right\| \leq \epsilon$ we obtain

$$
\alpha F\left(x_{1}\right)+(1-\alpha) F\left(x_{2}\right)-F\left(x_{0}\right)+k_{0} \in \operatorname{lin}(K)
$$


and

$$
\alpha f\left(\lambda, x_{1}\right)+(1-\alpha) f\left(\lambda, x_{2}\right)-f\left(\lambda, x_{0}\right)>-\lambda^{\top} k_{0} \geq-\epsilon\|\lambda\|
$$

for every $\lambda \in K^{*} \backslash K^{\perp}$. This shows using $\operatorname{lin}(K)=\left(K^{\perp}\right)^{\perp}$ that the function $f$ belongs to the set $\mathcal{A}$ with $P_{1}=K^{*} \backslash K^{\perp}$ and $P_{2}=K^{\perp}$. To prove the reverse implication we consider an arbitrary $k_{0} \in \operatorname{ri}(K)$. By the compactness of the set $K^{*} \cap \operatorname{lin}(K) \cap \partial B$ we obtain by relation (19) that

$$
2 \epsilon_{0}:=\inf \left\{\lambda^{\top} k_{0}: \lambda \in K^{*} \cap \operatorname{lin}(K) \cap \partial B\right\}>0 .
$$

Hence by (18) and $f$ belongs to $\mathcal{A}$ it follows for every $0<\alpha<1$ and $x_{1}, x_{2} \in D$ that there exists some $x_{0} \in D$ satisfying

$$
f\left(\lambda, x_{0}\right) \leq \alpha f\left(\lambda, x_{1}\right)+(1-\alpha) f\left(\lambda, x_{2}\right)+\epsilon_{0}\|\lambda\|
$$

for every $\lambda \in\left(K^{*} \cap \operatorname{lin}(K)\right) \backslash\{\mathbf{0}\}$ and

$$
\alpha f\left(\lambda, x_{1}\right)+(1-\alpha) f\left(\lambda, x_{2}\right)-f\left(\lambda, x_{0}\right)=0
$$

for every $\lambda \in K^{\perp}$. This yields by (27) and $\operatorname{lin}(K)=\left(K^{\perp}\right)^{\perp}$ that

$$
\alpha F\left(x_{1}\right)+(1-\alpha) F\left(x_{2}\right)-F\left(x_{0}\right) \in \operatorname{lin}(K) .
$$

Also by relations (25) and (26) we obtain

$$
\alpha f\left(\lambda, x_{1}\right)+(1-\alpha) f\left(\lambda, x_{2}\right)-f\left(\lambda, x_{0}\right)+\lambda^{\top} k_{0}>0
$$

for every $\lambda \in\left(K^{*} \cap \operatorname{lin}(K)\right) \backslash\{\mathbf{0}\}$. Applying to relations (28) and (29) the dual representation of $\operatorname{ri}(K)$ listed in (20) it follows that $\alpha F\left(x_{1}\right)+(1-\alpha) F\left(x_{2}\right)-F\left(x_{0}\right)+k_{0} \in \operatorname{ri}(K)$. Since $k_{0} \in \operatorname{ri}(K)$ is arbitrary, we finally obtain

$$
\alpha F(D)+(1-\alpha) F(D)+r i(K) \subseteq F(D)+r i(K),
$$

and by relation (14) the vector-valued function $F$ is $r i(K)$-convexlike.

If the convex cone $K \subseteq \mathbb{R}^{m}$ has a nonempty interior, then by relation (16) we may apply Theorem 12 to closely $K$-convexlike vector-valued functions. Moreover, since for $K$ full dimensional it follows that $K^{\perp}=\{\mathbf{0}\}$, the definition of the set $\mathcal{A}$ also simplifies. It is now easy to see for $K$ full dimensional that $F$ is closely $K$-convexlike if and only if for every $\epsilon>0,0<\alpha<1$ and $x_{1}, x_{2} \in D$ there exists some $x_{0} \in D$ satisfying

$$
f\left(\lambda, x_{0}\right) \leq \alpha f\left(\lambda, x_{1}\right)+(1-\alpha) f\left(\lambda, x_{2}\right)+\epsilon
$$

for every $\lambda \in K^{*} \backslash\{\mathbf{0}) \cap \partial B$. Note that the inequality listed in relation (30) is also known within minimax theory and called closely convex on $D$ (cf.[4]).

Definition 13 The bifunction $f: K^{*} \times D \rightarrow \mathbb{R}$ belongs to the class $\mathcal{B}$ if for every $\epsilon>0, x_{1}, x_{2}$ $\in D$ and $0<\alpha<1$ there exists some $x_{0} \in D$ and some $y_{0} \in \epsilon B$ satisfying

$$
f\left(\lambda, x_{0}\right) \leq \alpha f\left(\lambda, x_{1}\right)+(1-\alpha) f\left(\lambda, x_{2}\right)+\epsilon\|\lambda\|
$$

for every $\lambda \in P_{1}$ and

$$
f\left(\lambda, x_{0}\right)=\alpha f\left(\lambda, x_{1}\right)+(1-\alpha) f\left(\lambda, x_{2}\right)+\lambda^{\top} y_{0}
$$

for every $\lambda \in P_{2}$ with the sets $P_{1}, P_{2}$ a partition of $K^{*}$. 
By Theorem 12 and relation (16) the next result is only useful for a convex cone $K$ and a vector-valued function $F$ not satisfying $F(D) \subseteq x_{0}+\operatorname{lin}(K)$ for some $x_{0} \in$ aff $f(F(D))$.

Theorem 14 For $K \subseteq \mathbb{R}^{m}$ a nonempty convex cone it follows that $F: D \rightarrow \mathbb{R}^{m}$ is closely $K$ convexlike if and only if the function $f: K^{*} \times D \rightarrow \mathbb{R}$ defined in (21) belongs to the set $\mathcal{B}$ with $P_{1}=K^{*} \backslash K^{\perp}$ and $P_{2}=K^{\perp}$.

Proof. Let $F$ be closely $K$-convexlike and consider some arbitrary $\epsilon>0,0<\alpha<1$ and $x_{1}, x_{2} \in D$. By relation (15) and $\operatorname{cl}(F(D)+K) \subseteq F(D)+K+\epsilon B$ it follows that there exists some $x_{0} \in D, k_{0} \in K$ and $-y_{0} \in \epsilon B$ satisfying

$$
\alpha F\left(x_{1}\right)+(1-\alpha) F\left(x_{2}\right)=F\left(x_{0}\right)+k_{0}-y_{0} .
$$

This implies for every $\lambda \in K^{\perp}$ that

$$
\lambda^{\top} F\left(x_{0}\right)=\alpha \lambda^{\top} F\left(x_{1}\right)+(1-\alpha) \lambda^{\top} F\left(x_{2}\right)+\lambda^{\top} y_{0},
$$

while for every $\lambda \in K^{*} \backslash K^{\perp}$ and the Cauchy-Schwartz inequality we obtain

$$
\lambda^{\top} F\left(x_{0}\right) \leq \alpha \lambda^{\top} F\left(x_{1}\right)+(1-\alpha) \lambda^{\top} F\left(x_{2}\right)+\epsilon\|\lambda\| .
$$

Hence by (32) and (33) the function $f$ belongs to the set $\mathcal{B}$ with $P_{1}=K^{*} \backslash K^{\perp}$ and $P_{2}=K^{\perp}$. To prove the reverse implication it follows by (18) and $f$ belongs to $\mathcal{B}$ that for every $\epsilon>0,0<\alpha<1$ and $x_{1}, x_{2} \in D$ there exists some $x_{0} \in D$ and $y_{0} \in \epsilon B$ satisfying

$$
f\left(\lambda, x_{0}\right)-\alpha f\left(\lambda, x_{1}\right)-(1-\alpha) f\left(\lambda, x_{2}\right) \leq \epsilon
$$

for every $\lambda \in K^{*} \cap \operatorname{lin}(K) \cap \partial B$ and

$$
f\left(\lambda, x_{0}\right)=\alpha f\left(\lambda, x_{1}\right)+(1-\alpha) f\left(\lambda, x_{2}\right)+\lambda^{\top} y_{0}
$$

for every $\lambda \in K^{\perp}$. If $k_{0} \in \operatorname{ri}(K) \cap \partial B$ is fixed, we obtain by relation (19) that

$$
\epsilon_{0}:=\min \left\{\lambda^{\top} k_{0}: \lambda \in K^{*} \cap \operatorname{lin}(K) \cap \partial B\right\}>0 .
$$

This implies by (34), (36) and $y_{0} \in \epsilon B$ that

$$
f\left(\lambda, x_{0}\right)-\alpha f\left(\lambda, x_{1}\right)-(1-\alpha) f\left(\lambda, x_{2}\right)-\lambda^{\top}\left(y_{0}+3 \epsilon \epsilon_{0}^{-1} k_{0}\right)<0
$$

for every $\lambda \in K^{*} \cap \operatorname{lin}(K) \cap \partial B$. Since the function $\lambda \mapsto f(\lambda, x)$ is linear, it is obvious that relation (37) also holds for every $\lambda \in\left(K^{*} \cap \operatorname{lin}(K)\right) \backslash\{\mathbf{0}\}$. Also, by (35) and $k_{0} \in \operatorname{ri}(K)$ it follows that

$$
\alpha F\left(x_{1}\right)+(1-\alpha) F\left(x_{2}\right)-F\left(x_{0}\right)+y_{0}+3 \epsilon \epsilon_{0}^{-1} k_{0} \in \operatorname{lin}(K)+3 \epsilon \epsilon_{0}^{-1} k_{0} \subseteq \operatorname{lin}(K) .
$$

Applying to relations (37) and (38) the dual representation of $r i(K)$ listed in (20) yields

$$
\alpha F\left(x_{1}\right)+(1-\alpha) F\left(x_{2}\right)-F\left(x_{0}\right)+y_{0}+3 \epsilon \epsilon_{0}^{-1} k_{0} \in \operatorname{ri}(K) \subseteq K,
$$

and hence, using $y_{0} \in \epsilon B$ and $k_{0} \in \partial B$, we obtain

$$
\alpha F\left(x_{1}\right)+(1-\alpha) F\left(x_{2}\right) \in F(D)+K+\epsilon\left(1+3 \epsilon_{0}\right) B .
$$

Since $\epsilon>0$ is arbitrary, we may conclude that

$$
\alpha F(D)+(1-\alpha) F(D) \subseteq \operatorname{cl}(F(D)+K),
$$

and by (15) the vector-valued function $F$ is closely $K$-convexlike. 


\section{Conjugate functions, Lagrangian duality and cone convexlike vec- tor valued functions.}

In this section we will discuss conjugate functions, Lagrangian duality and the relation with the above class of cone convexlike vector-valued functions. In particular, we will first consider the lower semicontinuous hull $\bar{p}$ of a function $p: \mathbb{R}^{m} \rightarrow[-\infty, \infty]$ and show its relation with the biconjugate function $p^{* *}$ if $\bar{p}$ is convex. After having discussed these relations we will then introduce the primal problem $(P)$ already given in the introduction equipped with the Lagrangian perturbation scheme with perturbation parameter $y \in \mathbb{R}^{m}$, and show that the convexity of the associated lower semicontinuous hull $\bar{p}$ of the (Lagrangian perturbation) value function $p$ is convex if and only if some vector-valued function is closely convexlike. This enables us to apply the general results identified in the first part of this section and by doing so we derive under certain conditions, strong duality and related results for optimization problem $(P)$. In our analysis we need the following elementary result.

Lemma 15 If $C \subseteq \mathbb{R}^{m}$ is convex and $S \subseteq C$ is nonempty, then it follows that

$$
r i(C)) \subseteq S \Longleftrightarrow \operatorname{ri}(C)=\operatorname{ri}(S)
$$

Proof. The sufficiency part being trivial we only prove the necessary part. To verify this, let $r i(C)) \subseteq S$. Since the affine operator aff is monotone, $S \subseteq C$ and aff $(\operatorname{ri}(C))=\operatorname{aff}(C)$, we obtain aff $(C)=$ aff $f(S)$. This implies using $r i(C)) \subseteq S$ that $r i(C)=\operatorname{ri}(\operatorname{ri}(C)) \subseteq \operatorname{ri}(S)$. Since $S \subseteq C$ and aff $(C)=$ aff $(S)$ we also have $\operatorname{ri}(S) \subseteq \operatorname{ri}(C)$, and the result is proved.

To continue our analysis let $p: \mathbb{R}^{m} \rightarrow[-\infty, \infty]$ be an extended real-valued function with effective domain $\operatorname{dom}(p):=\left\{x \in \mathbb{R}^{m}: p(x)<\infty\right\}$ nonempty and let $\bar{p}$ denote the lower semicontinuous hull of the function $p$. It is well-known (cf.[17], [6]) that $\operatorname{epi}(\bar{p})=\operatorname{cl}(\operatorname{epi}(p))$ with $e p i(p):=e p i_{\mathbb{R}_{+}}(p)$ denoting the epigraph of the function $p$ and

$$
\operatorname{dom}(p) \subseteq \operatorname{dom}(\bar{p}) \subseteq \operatorname{cl}(\operatorname{dom}(p)) .
$$

Applying now relation (39) we immediately obtain

$$
c l(\operatorname{dom}(p))=\operatorname{cl}(\operatorname{dom}(\bar{p})) \text { and } \operatorname{aff}(\operatorname{dom}(p))=\operatorname{aff}(\operatorname{dom}(\bar{p})) .
$$

Also by Lemma 15 and relation (39) it follows for $\operatorname{dom}(\bar{p})$ convex that

$$
\operatorname{ri}(\operatorname{dom}(\bar{p})) \subseteq \operatorname{dom}(p) \Longleftrightarrow \operatorname{ri}(\operatorname{dom}(\bar{p}))=\operatorname{ri}(\operatorname{dom}(p)) .
$$

If we denote by $p^{*}(\lambda):=\sup \left\{\lambda^{\top} y-p(y): y \in \mathbb{R}^{m}\right\}$ the conjugate function of the function $p$ and $p^{* *}$ its biconjugate function the next corollary is an easy consequence of the Fenchel-Moreau theorem and some well-known function description of $\bar{p}$.

Corollary 16 If the lower semicontinuous hull $\bar{p}$ of a function $p: \mathbb{R}^{m} \rightarrow[-\infty, \infty]$ is convex, then it follows for every $y_{0}$ belonging to dom $(\bar{p})$ that $p^{* *}\left(y_{0}\right)=\bar{p}\left(y_{0}\right)=\liminf _{y \rightarrow y_{0}} p(y)$.

Proof. Since $y_{0}$ belongs to $\operatorname{dom}(\bar{p})$ it follows that $\bar{p}\left(y_{0}\right)$ is finite or $\bar{p}\left(y_{0}\right)=-\infty$. If $\bar{p}\left(y_{0}\right)$ is finite the convex function $\bar{p}$ is proper and by the Fenchel Moreau theorem (cf.[17], [6]) we obtain $p^{* *}\left(y_{0}\right)=\bar{p}\left(y_{0}\right)$. Moreover, if $\bar{p}\left(y_{0}\right)=-\infty$, it follows by contradiction that $p^{*}$ is identically $\infty$ 
and so $p^{* *}\left(y_{0}\right) \equiv-\infty$. Hence we obtain in both cases that $p^{* *}\left(y_{0}\right)=\bar{p}\left(y_{0}\right)$ and since $\bar{p}\left(y_{0}\right)=$ $\liminf _{y \rightarrow y_{0}} p(y)$ (cf.[17], [6]) the result follows.

Up to now we did not show that the optimization problem associated with the biconjugate function $p^{* *}$ has an optimal solution. The next corollary is an immediate consequence of some standard results in convex analysis.

Corollary 17 If the lower semicontinuous hull $\bar{p}$ of a function $p: \mathbb{R}^{m} \rightarrow[-\infty, \infty]$ is convex, then it follows for every $y_{0}$ belonging to ri $(\operatorname{dom}(\bar{p}))$ that there exists some $\lambda_{0} \in \mathbb{R}^{m}$ satisfying $p^{* *}\left(y_{0}\right)=y_{0}^{\top} \lambda_{0}-p^{*}\left(\lambda_{0}\right)=\bar{p}\left(y_{0}\right)=\liminf _{y \rightarrow y_{0}} p(y)$.

Proof. If $\bar{p}\left(y_{0}\right)=-\infty$ we know $p^{*} \equiv \infty$, and the result is obvious. For $\bar{p}\left(y_{0}\right)$ finite and $y_{0}$ belongs to $\operatorname{ri}(\operatorname{dom}(\bar{p}))$ the subgradient set $\partial \bar{p}\left(y_{0}\right)$ is nonempty (cf.[17], [6]) and

$$
\lambda \in \partial \bar{p}\left(y_{0}\right) \Longleftrightarrow \bar{p}^{*}(\lambda)=\lambda^{\top} y_{0}-\bar{p}\left(y_{0}\right) .
$$

Applying now Corollary 16, $p^{*} \leq \bar{p}^{*}$ and relation (42), it follows for $\lambda_{0}$ belonging to $\partial \bar{p}\left(y_{0}\right)$ that $\bar{p}\left(y_{0}\right)=p^{* *}\left(y_{0}\right) \geq\left(\bar{p}^{*}\right)^{*}\left(y_{0}\right) \geq y_{0}^{\top} \lambda_{0}-\bar{p}^{*}\left(\lambda_{0}\right)=\bar{p}\left(y_{0}\right)$, and the result is proved.

To replace in Corollary 17 the value $\liminf _{y \rightarrow y_{0}} p(y)$ by $p\left(y_{0}\right)$ for $y_{0}$ belonging to $\operatorname{ri}(\operatorname{dom}(\bar{p}))$, it is necessary and sufficient to assume that $p$ is lower semicontinuous in $y_{0}$, or equivalently $\bar{p}\left(y_{0}\right)=p\left(y_{0}\right)$. To achieve this, we introduce the following class of functions.

Definition 18 A function $p: \mathbb{R}^{m} \rightarrow[-\infty, \infty]$ is called almost convex if the lower semicontinuous hull $\bar{p}$ of $p$ is convex and ri $($ epi $(\bar{p}))$ is a subset of epi $(p)$.

In the next result we give an equivalent description of an almost convex function.

Theorem 19 For any function $p: \mathbb{R}^{m} \rightarrow[-\infty, \infty]$ with dom $(p)$ nonempty, it follows that

$$
p \text { almost convex } \Longleftrightarrow \bar{p} \text { convex and } \bar{p}(y)=p(y) \text { for every } y \in \operatorname{ri}(\operatorname{dom}(\bar{p})) \text {. }
$$

Proof. If $p$ is an almost convex function with $\operatorname{dom}(p)$ nonempty, we only need to show that $\bar{p}(y)=p(y)$ for every $y \in \operatorname{ri}(\operatorname{dom}(\bar{p}))$. By relation (39) the set $\operatorname{dom}(\bar{p})$ is also nonempty. Since it is well-known (cf.[17], [6]) that

$$
\operatorname{ri}(\operatorname{epi}(\bar{p}))=\{(y, r): \bar{p}(y)<r, y \in \operatorname{ri}(\operatorname{dom}(\bar{p})\}
$$

and by assumption $\operatorname{ri}(\operatorname{epi}(\bar{p})) \subseteq \operatorname{epi}(p)$, it follows for every $y \in \operatorname{ri}(\operatorname{dom}(\bar{p}))$ and $\epsilon>0$ that $(y, \bar{p}(y)+\epsilon)$ belongs to $\operatorname{epi}(p)$. This implies $\bar{p}(y) \geq p(y)$ for every $y \in \operatorname{ri}(\operatorname{dom}(\bar{p}))$ and hence $\bar{p}$ equals $p$ on $\operatorname{ri}(\operatorname{dom}(\bar{p}))$. To prove the reverse implication, let $\bar{p}$ convex and $\bar{p}(y)=p(y)$ for every $y \in \operatorname{ri}(\operatorname{dom}(\bar{p}))$. This implies $\operatorname{ri}(\operatorname{dom}(\bar{p})) \subseteq \operatorname{dom}(p)$ and by relation (41) we obtain $\operatorname{ri}(\operatorname{dom}(\bar{p}))=\operatorname{ri}(\operatorname{dom}(p))$. Hence by the representation of the relative interior of the epigraph of a convex function (see relation (43)) and the previous observations it follows that

$$
r i(\operatorname{epi}(\bar{p}))=\{(y, r): p(y)<r, y \in \operatorname{ri}(\operatorname{dom}(p)\} \subseteq \operatorname{epi}(p),
$$

and so $p$ is almost convex.

As shown in the above proof it follows for $p$ almost convex with $\operatorname{dom}(p)$ nonempty that

$$
\operatorname{ri}(\operatorname{dom}(p))=\operatorname{ri}(\operatorname{dom}(\bar{p}))
$$

and these sets are nonempty. The next result is an immediate consequence of Corollary 17, Theorem 19 and relation (44). 
Corollary 20 If the function $p: \mathbb{R}^{m} \rightarrow[-\infty, \infty]$ is almost convex with dom $(p)$ nonempty, then it follows for every $y_{0}$ belonging to $\mathrm{ri}(\operatorname{dom}(p))$ that there exists some $\lambda_{0} \in \mathbb{R}^{m}$ satisfying $p^{* *}\left(y_{0}\right)=$ $y_{0}^{\top} \lambda_{0}-p^{*}\left(\lambda_{0}\right)=p\left(y_{0}\right)$.

To see what happens for $p$ almost convex if $y_{0}$ does not belong to $\operatorname{ri}(\operatorname{dom}(p))$, but to the relative boundary $\operatorname{rbd}(\operatorname{dom}(p))$ given by

$$
\operatorname{rbd}(\operatorname{dom}(p)):=\operatorname{cl}(\operatorname{dom}(p)) \backslash \operatorname{ri}(\operatorname{dom}(p)),
$$

we first need the following observation. By relations (40) and (44) we obtain for $p$ almost convex and $\operatorname{dom}(p)$ nonempty that

$$
y_{0}+t\left(y_{1}-y_{0}\right) \in \operatorname{ri}(\operatorname{dom}(p))
$$

for every $y_{0} \in \operatorname{cl}(\operatorname{dom}(p)), y_{1} \in \operatorname{ri}(\operatorname{dom}(p))$ and $0<t<1$. It is now possible to show the following result for an almost convex function $p$ with nonempty effective domain using some standard results from convex analysis, Theorem 19 and relation (45).

Corollary 21 If the function $p: \mathbb{R}^{m} \rightarrow[-\infty, \infty]$ is almost convex with dom $(p)$ nonempty, then it follows for every $y_{0}$ belonging to $\operatorname{rbd}(\operatorname{dom}(p))$ and $y_{1}$ belonging to ri $(\operatorname{dom}(p))$ that $p^{* *}\left(y_{0}\right)=$ $\lim _{t \downarrow 0} p\left(y_{0}+t\left(y_{1}-y_{0}\right)\right)$.

Proof. Since $\operatorname{dom}(p)$ is nonempty it follows by the observation after relation (44) that $r i(\operatorname{dom}(p))$ is nonempty. If for every $y \in \operatorname{ri}(\operatorname{dom}(p))$ it holds that $p(y)=-\infty$, we obtain that $p^{* *}\left(y_{0}\right)=-\infty$. This implies by relation (45) the desired result and so we still need to prove the result if there exists some $y \in \operatorname{ri}(\operatorname{dom}(p))$ with $p(y)$ finite. This implies by Theorem 19 that $\bar{p}(y)=p(y)$ is finite and hence $\bar{p}$ is a proper convex function. Applying now the Fenchel-Moreau theorem yields $p^{* *}=\bar{p}$ and this shows that $p^{* *}$ is a lower semicontinuous proper convex function. Since $p^{* *}=\bar{p}$, it also follows using relation (44) that

$$
\operatorname{ri}\left(\operatorname{dom}\left(p^{* *}\right)\right)=\operatorname{ri}(\operatorname{dom}(\bar{p}))=\operatorname{ri}(\operatorname{dom}(p))
$$

and this implies (cf.[17], [6]) for every $y_{1} \in \operatorname{ri}(\operatorname{dom}(p))$ and $y_{0} \in \operatorname{rbd}(\operatorname{dom}(p))$ that

$$
p^{* *}\left(y_{0}\right)=\lim _{t \downarrow 0} p^{* *}\left(y_{0}+t\left(y_{1}-y_{0}\right)\right) .
$$

By relation (45) and Corollary 20 we obtain

$$
p^{* *}\left(y_{0}+t\left(y_{1}-y_{0}\right)\right)=p\left(y_{0}+t\left(y_{1}-y_{0}\right)\right)
$$

for every $0<t<1$ and by relations (47) and (48) the desired result follows.

In what follows we will apply the previous results to the Lagrangian perturbation scheme of the optimization problem $(P)$. Consider the value function $p: \mathbb{R}^{m} \rightarrow[-\infty, \infty]$ given by

$$
p(y):=\inf \left\{f(x): F(x) \leq_{K} y, x \in D\right\} .
$$

Clearly for $y=\mathbf{0}$ we recover our initial optimization problem $(P)$ and it is easy to see that $\operatorname{dom}(p)=F(D)+K$. Since we always assume that the feasible region $\mathcal{F}$ is nonempty, or equivalently $\mathbf{0} \in F(D)+K$ we obtain $\mathbf{0} \in \operatorname{dom}(p)$. Introducing for the perturbed problem the feasible region $\mathcal{F}(y)$ given by

$$
\mathcal{F}(y):=\{x: F(x) \in-K+y, x \in D\}
$$


it is follows by definition for $\mathcal{F}(y)$ empty that $p(y)=\infty$. If the vector-valued function $H: D \longrightarrow$ $\mathbb{R}^{m+1}$ is given by

$$
H(x)=(F(x), f(x))
$$

then the next result is easy to show.

Theorem 22 It follows that the function $\bar{p}$ is convex if and only if the function $H$ is closely $K \times$ $\mathbb{R}_{+}$-convexlike. Moreover, the function $p$ is convex if and only the function $H$ is $K \times \operatorname{int}\left(\mathbb{R}_{+}\right)$convexlike.

Proof. If epi $i_{s}(p):=\operatorname{epi}_{\text {int }\left(\mathbb{R}_{+}\right)}(p)$ denotes the strict epigraph of $p$, then it is easy to verify that

$$
\operatorname{epi}(\bar{p})=\operatorname{cl}(\operatorname{epi}(p))=\operatorname{cl}\left(e p i_{s}(p)\right) .
$$

By relation (49) we obtain

$$
e p i_{s}(p)=\left\{(y, r): \exists_{x \in D} y \in F(x)+K \text { and } f(x)<r\right\}=H(D)+\left(K \times \operatorname{int}\left(\mathbb{R}_{+}\right)\right),
$$

and this shows by relations (51) that $\operatorname{epi}(\bar{p})=\operatorname{cl}\left(H(D)+\left(K \times \mathbb{R}_{+}\right)\right)$. To verify the second part, it follows by Theorem 8 that $e p i(p)$ is convex if and only if $e p i_{s}(p)$ is convex. Applying relation (52) yields the desired result.

The next result is well-known and can be easily verified.

Lemma 23 If the function $\theta: K^{*} \rightarrow[-\infty, \infty)$ is the Lagrangian function

$$
\theta(\lambda):=\inf \left\{f(x)+\lambda^{\top} F(x): x \in D\right\}
$$

then it follows with $p$ given by relation (49) that

$$
-p^{*}(-\lambda)= \begin{cases}\theta(\lambda) & \lambda \in K^{*} \\ -\infty & \text { otherwise. }\end{cases}
$$

By Lemma 23 we obtain that $p^{* *}(y)=\sup \left\{-\lambda^{\top} y+\theta(\lambda): \lambda \in K^{*}\right\}$. This yields for $y=\mathbf{0}$ that

$$
p^{* *}(\mathbf{0})=\sup \left\{\theta(\lambda): \lambda \in K^{*}\right\}=v(D) .
$$

As already observed in the introduction, the above problem is called the Lagrangian dual problem $(D)$. We are now ready to prove the following main results for the primal optimization problem $(P)$ and its Lagrangian dual problem.

Theorem 24 If 0 belongs to $F(D)+K$ and the vector-valued function $H: D \rightarrow \mathbb{R}^{m+1}$, listed in relation (50), is closely $K \times \mathbb{R}_{+}$-convexlike, then it follows that

$$
v(D)=\liminf _{y \rightarrow 0} p(y)
$$

with $p$ given by relation (49). Also the function $p$ is lower semicontinuous at $\mathbf{0}$ if and only if $v(D)=p(\mathbf{0})$. Finally, if $\mathbf{0}$ belongs to $r i(F(D)+K)$, then it follows that the dual problem $(D)$ has an optimal solution. 
Proof. By Theorem 22 we know that the vector-valued function $H$ is closely $K \times \mathbb{R}_{+}$-convexlike if and only if the function $\bar{p}$ is convex. Moreover, by relations (39) and $\operatorname{dom}(p)=F(D)+K$ it follows that

$$
\mathbf{0} \in F(D)+K \subseteq \operatorname{dom}(\bar{p}) .
$$

This yields by Corollary 16 that $v(D)=p^{* *}(\mathbf{0})=\liminf _{y \rightarrow 0} p(y)$. Also it holds that $\liminf _{y \rightarrow 0} p(y)=p(\mathbf{0})$ if and only if $p$ is lower semicontinuous at $\mathbf{0}$ and using this in combination with the first part, the second part follows. To verify the last part, we observe by relation (39) and the observation after this relation that $\mathbf{0}$ belongs to $\operatorname{ri}(F(D)+K)=\operatorname{ri}(\operatorname{dom}(p)) \subseteq \operatorname{ri}(\operatorname{dom}(\bar{p}))$. This implies by Corollary 17 and relation (53) that the dual problem has an optimal solution.

Discussing a more general parametric optimization problem, in [15] a geometrical interpretation is given for $p$ lower semicontinuous at $\mathbf{0}$. In case we impose the additional regularity condition

$$
\operatorname{ri}\left(\operatorname{cl}\left(H(D)+\left(K \times \mathbb{R}_{+}\right)\right)\right) \subseteq H(D)+\left(K \times \mathbb{R}_{+}\right),
$$

then the following improvement of Theorem 24 can be verified. It will be shown that this additional condition is always satisfied if the vector-valued function $H$ is $r i(K) \times \operatorname{int}\left(\mathbb{R}_{+}\right)$-convexlike.

Theorem 25 If 0 belongs to $F(D)+K$ and the vector-valued function $H: D \rightarrow \mathbb{R}^{m+1}$ is closely $K \times \mathbb{R}_{+}$-convexlike and satisfies relation (56), then it follows that $v(D)=\lim _{t \downarrow 0} p\left(t y_{1}\right)$ for every $y_{1}$ belonging $\mathrm{ri}(F(D)+K)$. If $\mathbf{0}$ belongs to ri $(F(D)+K)$, then the dual problem has an optimal solution and $v(D)=p(\mathbf{0})$.

Proof. By relation (56) and Theorem 22 we obtain that the function $p$ is almost convex. Hence the result follows from Corollaries 20 and 21.

By Theorem 2.2 of [5] it follows for any nonempty convex cone $K$ that

$$
\operatorname{cl}\left(H(D)+\left(K \times \mathbb{R}_{+}\right)\right)=\operatorname{cl}\left(H(D)+\left(\operatorname{ri}(K) \times \operatorname{int}\left(\mathbb{R}_{+}\right)\right)\right) .
$$

This shows for $H: D \rightarrow \mathbb{R}^{m+1}$ a $\operatorname{ri}(K) \times \operatorname{int}\left(\mathbb{R}_{+}\right)$-convexlike vector-valued function that

$$
\begin{aligned}
\operatorname{ri}\left(\operatorname{cl}\left(H(D)+\left(K \times \mathbb{R}_{+}\right)\right)\right) & =\operatorname{ri}\left(\operatorname{cl}\left(H(D)+\left(\operatorname{ri}(K) \times \operatorname{int}\left(\mathbb{R}_{+}\right)\right)\right)\right. \\
& =\operatorname{ri}\left(H(D)+\left(\operatorname{ri}(K) \times \operatorname{int}\left(\mathbb{R}_{+}\right)\right)\right) .
\end{aligned}
$$

Hence for $\mathrm{H}$ a $\operatorname{ri}(K) \times \operatorname{int}\left(\mathbb{R}_{+}\right)$-convexlike vector-valued function we obtain that the regularity condition given by relation (56) automatically holds. Finally we like to observe that the second part of Theorem 25 is also verified in [5] by means of the image space approach of Giannessi and in the same paper it is shown that the condition of Theorem 25 is still weaker than the assumption that $H$ is $\operatorname{ri}(K) \times \operatorname{int}\left(\mathbb{R}_{+}\right)$-convexlike.

\section{Appendix}

In this appendix we show a technical result needed in Section 1. To show this result we first need the following lemma.

Lemma 26 For $S \subseteq \mathbb{R}^{m}$ and $K \subseteq \mathbb{R}^{m}$ a nonempty convex cone it follows that there exists some $x_{0} \in \operatorname{aff}(S)$ satisfying $S \subseteq x_{0}+\operatorname{lin}(K)$ if and only if for every $x \in$ aff $(S+K)$ it holds that aff $(S+K)=x+\operatorname{lin}(K)$. 
Proof. Let aff $(S)=x+\operatorname{lin}(K)$ for every $x \in \operatorname{aff}(S+K)$. Since $\mathbf{0} \in \operatorname{cl}(K)$ we obtain $S \subseteq \operatorname{aff}(S) \subseteq$ aff $(S+\operatorname{cl}(K))=$ af $f(S+K)$ and hence by our assumption there exists some $x_{0} \in \operatorname{aff}(S)$ satisfying $S \subseteq$ aff $(S)=x_{0}+\operatorname{lin}(K)$. To show the reverse implication we first observe for every $x \in \operatorname{aff}(S+K)$ that $x+\operatorname{lin}(K) \subseteq$ aff $(S+K)+\operatorname{lin}(K)$. Since $K$ is a convex cone this yields (cf.[6]) that

$$
\operatorname{aff}(S+K)+\operatorname{lin}(K)=\operatorname{aff}(S+K)+\operatorname{aff}(K)=\operatorname{aff}(S+K+K)=\operatorname{aff}(S+K),
$$

and hence $x+\operatorname{lin}(K) \subseteq$ aff $f(S+K)$ for every $x \in$ aff $f(S+K)$. To show the reverse inclusion we need our assumption $S \subseteq x_{0}+\operatorname{lin}(K)$ for some $x_{0} \in$ aff $(S)$. Using this assumption we obtain $S+K \subseteq \operatorname{lin}(K)+K+x_{0} \subseteq \operatorname{lin}(K)+x_{0}$ and this shows

$$
\operatorname{aff}(S+K) \subseteq \operatorname{lin}(K)+x_{0}
$$

It is well-known in linear algebra (cf.[12]) that af $f(S+K)=\operatorname{lin}(S+K-x)+y$ for every $x, y \in$ aff $f(S+K)$. This implies in combination with relation (58) that af $f(S+K) \subseteq \operatorname{lin}(K)+x$ for every $x \in$ af $f(S+K)$ and this shows the desired result.

Using Lemma 26 one can show the following theorem. This result generalizes a similar result proved by Breckner and Kassay (cf.[1]) for convex cones having a nonempty interior. If the cone $K \subseteq \mathbb{R}^{m}$ has a nonempty interior, then $\operatorname{lin}(K)=\mathbb{R}^{m}$ and so the condition in the next theorem is automatically satisfied.

Theorem 27 Let $K \subseteq \mathbb{R}^{m}$ be a nonempty convex cone and $S \subseteq \mathbb{R}^{m}$ some set satisfying $S \subseteq$ $x_{0}+\operatorname{lin}(K)$ for some $x_{0} \in$ af $f(S)$. Then it follows that $\operatorname{ri}(\operatorname{cl}(S+K))=S+\operatorname{ri}(K)$.

Proof. We first show that $S+\operatorname{ri}(K) \subseteq \operatorname{ri}(\operatorname{cl}(S+K))$. If $x$ belongs to $S+\operatorname{ri}(K)$, it follows that there exist some $s \in S$ and $k \in \operatorname{ri}(K) \subseteq K$ satisfying $x=s+k$. Clearly $x$ belongs to aff $f(S+K)$ and since $k$ belongs to $\operatorname{ri}(K)$ and aff $f(K)=\operatorname{lin}(K)$ for any convex cone, one can find some $\epsilon_{0}>0$ satisfying

$$
\left(k+\epsilon_{0} B\right) \cap \operatorname{lin}(K) \subseteq K
$$

with $B:=\left\{x \in \mathbb{R}^{m}:\|x\| \leq 1\right\}$ denoting the closed Euclidean unit ball. By Lemma 26 and $s \in S \subseteq$ aff $f(S+K)$ we obtain aff $(S+K)=s+\operatorname{lin}(K)$. This implies in combination with relation (59) that

$$
\left(x+\epsilon_{0} B\right) \cap \operatorname{aff}(S+K)=\left(s+k+\epsilon_{0} B\right) \cap(s+\operatorname{lin}(K)) \subseteq s+K,
$$

and so $x$ belongs to $\operatorname{ri}(\operatorname{cl}(S+K)$. To verify the inclusion $\operatorname{ri}(\operatorname{cl}(S+K)) \subseteq S+\operatorname{ri}(K)$, consider some $x \in \operatorname{ri}(\operatorname{cl}(S+K))$. By definition there exists some $\epsilon_{1}>0$ satisfying

$$
\left(x+\epsilon_{1} B\right) \cap \operatorname{aff}(S+K)=\left(x+\epsilon_{1} B\right) \cap \operatorname{aff}(\operatorname{cl}(S+K)) \subseteq \operatorname{cl}(S+K) .
$$

Since $\operatorname{ri}(K)$ is a nonempty convex cone, there exists some $k_{0} \in \operatorname{ri}(K)$ with $\left\|k_{0}\right\| \leq \frac{\epsilon_{1}}{2}$ and this yields $x-k_{0} \in x+\epsilon_{1} B$. Moreover, since aff $\left(S_{1}\right)+\operatorname{aff}\left(S_{2}\right)=\operatorname{aff}\left(S_{1}+S_{2}\right)$ for every $S_{i} \subseteq \mathbb{R}^{m}$ it follows that

$$
x-k_{0} \in \operatorname{aff}(S+K)-\operatorname{aff}(K)=\operatorname{aff}(S+K)
$$

and this implies by relation (60) that the point $x-k_{0}$ belongs to $c l(S+K)$, or equivalently,

$$
\left(x-k_{0}+\epsilon B\right) \cap(S+K) \neq \emptyset
$$


for every $\epsilon>0$. At the same time, due to $k_{0}$ belongs to $\operatorname{ri}(K) \subseteq K$, there exists some $\epsilon_{2}>0$ satisfying

$$
\left.\left(k_{0}+\epsilon_{2} B\right) \cap \operatorname{linK}\right) \subseteq \operatorname{ri}(K),
$$

while for $x$ belonging to $\operatorname{cl}(S+K)$ we know by Lemma 26 that

$$
\text { aff }(S+K)=x+\operatorname{lin}(K)=x-\operatorname{lin}(K) .
$$

Combining relations (62) and (63) yields

$$
\left(x-k_{0}+\epsilon_{2} B\right) \cap \operatorname{aff}(S+K)=x-\left(\left(k_{0}+\epsilon_{2} B\right) \cap \operatorname{lin}(K)\right) \subseteq x-\operatorname{ri}(K) .
$$

Since by relation (61) one can find some $s \in S$ and $k \in K$ satisfying

$$
s+k \in\left(x-k_{0}+\epsilon_{2} B\right)
$$

this implies by relation (64) that $s+k \in x-\operatorname{ri}(K)$. Hence it follows that $x$ belongs to $s+k+$ $r i(K) \subseteq S+r i(K)$ and so we have verified that $r i(\operatorname{cl}(S+K)) \subseteq S+\operatorname{ri}(K)$

Acknowledgements. The authors like to thank the comments of the anonymous referees and the editor for their remarks and suggestions, which greatly improved the presentation of this paper.

\section{References}

[1] Breckner, W.W. and G., Kassay. A systematization of convexity concepts for sets and functions. Journal of Convex Analysis, 4:1-19, 1997.

[2] Cambini, R and S., Komlósi. On the scalarization of pseudoconcavity and pseudomonotonicity concepts for vector valued functions. In Crouzeix, J.P., Martinez-Legaz, J.E. and M., Volle, editor, Generalized Convexity, Generalized Monotonicity, pages 277-283. Kluwer Academic Publishers, 1998.

[3] Fan, K. Minimax theorems. Proc.Nat.Acad.Sci.U.S.A., 39:42-47, 1953.

[4] Frenk, J.B.G. and G., Kassay. Minimax results and finite dimensional separation. Journal of Optimzation Theory and Applications, 113(2):409-421, 2002.

[5] Frenk, J.B.G and G. Kassay. On classes of generalized convex functions, Gordan-Farkas type theorems and Lagrangian duality. Journal of Optimization Theory and Applications, 102(2):315-343, 1999.

[6] Frenk, J.B.G. and G. Kassay. Introduction to convex and quasiconvex analysis. In Hadjisavvas, N., Komlósi, S. and S. Schaible, editor, Handbook of Generalized Convexity and Generalized Monotonicity, pages 3-87. Springer, Dordrecht, 2005.

[7] Frenk, J.B.G., Kassay, G. and J.Kolumbán. On equivalent results in minimax theory. European Journal of Operational Research, 157:46-58, 2004.

[8] Giannessi, F. Theorems of the alternative and optimality conditions. Journal of Optimization Theory and Applications, 42:331-365, 1984. 
[9] Hayashi, M. and H., Komiya. Perfect duality for convexlike programs. Journal of Optimization Theory and Applications, 38:179-189, 1982.

[10] Jeyakumar, V. A generalization of a minimax theorem of Fan via a theorem of the alternative. Journal of Optimization Theory and Applications, 48:525-533, 1986.

[11] Kreyszig, E. Introductory Functional Analysis with Applications. Wiley, New York, 1978.

[12] Lancaster, P. and M., Tismenetsky. The Theory of Matrices (second edition). Academic Press, New York, 1985.

[13] Li, Z.P. and S.Y., Wang. Lagrange multipliers and saddlepoints in multiobjective programming. Journal of Optimization Theory and Applications, 83, 1994.

[14] Mastroeni, G. and T., Rapcsák. On convex generalized systems. Journal of Optimization Theory and Applications, 104(2):605-627, 2000.

[15] Mastroeni, G., Pappalardo, M. and N.D., Yen. Image of a parametric optimization problem and continuity of the perturbation function. Journal of Optimization Theory and Applications, 81:193-202, 1994.

[16] Paeck, S. Convexlike and concavelike conditions in alternative, minmax and minimization theorems. Journal of Optimization Theory and Applications, 74:317-332, 1992.

[17] Rockafellar, R.T. Convex Analysis. Princeton University Press, Princeton, 1970.

[18] Rockafellar, R.T. Conjugate duality and optimization. SIAM, Philadelphia, 1974.

[19] Rudin, W. Functional Analysis. McGraw-Hill, New York, 1991.

[20] Sturm, J.F. Theory and algorithms of semidefinite programming. In Hans Frenk, Kees Roos, Tamás Terlaky and Shuzhong Zhang, editor, High Performance Optimization, volume 33 of Applied Optimization, pages 3-194, Dordrecht, 2000. Kluwer Academic Publishers.

[21] Yu, P.L. Cone extreme points and nondominated solutions in decision problems with multiobjectives. Journal of Optimization Theory and Applications, 14(3):319-377, 1974. 


\section{Publications in the Report Series Research* in Management}

\section{ERIM Research Program: "Business Processes, Logistics and Information Systems"}

\section{5}

On The Design Of Artificial Stock Markets

Katalin Boer, Arie De Bruin And Uzay Kaymak

ERS-2005-001-LIS

http://hdl.handle.net/1765/1882

Knowledge sharing in an Emerging Network of Practice: The Role of a Knowledge Portal Peter van Baalen, Jacqueline Bloemhof-Ruwaard, Eric van Heck

ERS-2005-003-LIS

A note on the paper Fractional Programming with convex quadratic forms and functions by H.P.Benson J.B.G.Frenk

ERS-2005-004-LIS

A note on the dual of an unconstrained (generalized) geometric programming problem

J.B.G.Frenk and G.J.Still

ERS-2005-006-LIS

A Modular Agent-Based Environment for Studying Stock Markets

Katalin Boer, Uzay Kaymak and Arie de Bruin

ERS-2005-17-LIS

Keywords Lagrangian duality, cone convexlike functions

J.B.G. Frenk and G. Kassay

ERS-2005-019-LIS

* A complete overview of the ERIM Report Series Research in Management: https://ep.eur.nl/handle/1765/1

ERIM Research Programs:

LIS Business Processes, Logistics and Information Systems

ORG Organizing for Performance

MKT Marketing

F\&A Finance and Accounting

STR Strategy and Entrepreneurship 